\title{
Ca2+-Camk-CREB Mediates the Effect of Flavonoids from Scutellaria Baicalensis Stems and Leaves on the Decrease of Neuroregeneration-Induced by Composited A $\beta$ in Rats
}

\author{
Zhang Hui ${ }^{1}$, Ding Shengkai ${ }^{1}$, Liu Qianqian ${ }^{1}$, Ye Yuanyuan $^{1}$, Xu Qian ${ }^{1}$, \\ Shang Yazhen ${ }^{1,2 *}$ \\ ${ }^{1}$ Institute of Traditional Chinese Medicine, Chengde Medical College; Key Research Laboratory of Anti- \\ dementia of Traditional Chinese Medicine, Hebei Province; Key Laboratory of Traditional Chinese Medicine \\ Research and Development of Hebei Province; Institute of Basic Medicine, Chengde Medical College; Hebei \\ Chengde 067000, China. \\ ${ }^{2}$ Chengde Medical College Shang Yazhen Anyuan Road, Institute of Traditional Chinese Medicine, Chengde \\ Medical College 067000 Chengde China \\ *Corresponding Author: Shang Yazhen, Institute of Traditional Chinese Medicine, Chengde Medical \\ College, Chengde 067000; The Fourth Hospital of Shijiazhuang, Shijiazhuang, Hebei 050011, PR China.
}

\begin{abstract}
:
Objective

To investigate the effects of Scutellaria baicalensis stem-leaf flavonoid (SSF) on the decrease of neuroregeneration of rats induced by $A \beta_{25-35}$ in combination with AlCl3 and RHTGF- $\beta 1$ (composited A $\beta$ ) and its regulatory mechanism.

Methods

The healthy adult male Sprague Dawley (SD) rats were microinjected composited A into cerebral ventricle to establish the model of mimic Alzheimer's disease (AD). After 45 days of operation, Morris water maze was used to screen the memory impairment model, and the successful model rats were randomly divided into model group and three-doses of drug groups. Rats in the drug group were treated SSF by intragastric administration (ig) for 30 days, and the model group and the sham group rats were treated with the equal volume saline. Immunohistochemistry was used to detect the expression of NeuN protein in the hippocampal gyrus of rats, and the expression levels of CaMKII, P-CREB-Ser133, FGF2, Egr-1 protein and mRNA expression levels of CaMKII, FGF2 and Egr-1 in the hippocampus and cerebral cortex of rats were detected by Western blotting and Quantitative real-time polymerase chain reaction ( $q P C R)$.
\end{abstract}

\section{Results}

Immunohistochemistry showed that the protein expression of NeuN in the hippocampal gyrus of the model group rats was significantly decreased, as compared with the sham group. Compared with the model group, three-doses of SSF can significantly increase the potein expression of NeuN in the hippocampal gyrus than that of model group. Compared with the sham group, the protein expressions levels of CaMKII, P-CREB-Ser133, FGF2 and Egr-1 by Western blotting detected were dramatically lowered, and the mRNA expression levels of CaMKII, FGF2 and Egr-1 by qPCR assayed showed differently alteration of hippocampus and cerebral cortex in model group. While SSF differently regulated the changes of CaMKII, P-CREB-Ser133, FGF2, Egr-1 proteins and CaMKII, FGF2, Egr-1 mRNA expressions in rats' hippocampus and cerebral cortex induced by composited A . Conclusion: Intraventricular injection of composited A $\beta$ can reduce the neuroregeneration, and CaMKII, P-CREB-Ser133, FGF2 and Egr-1 proteins and CaMKII, FGF2 and Egr-1 mRNA expression in the $\mathrm{Ca}^{2+}$-CaMK-CREB signaling pathway change. While SSF can reverse the decrease of neuroregeneration induced by composited $A \beta$, and the effective mechanism of SSF may be primarily from the regulation of SSF in CaMKII, p-CREB-Ser133, FGF2 and Egr-1 expressions in the $\mathrm{Ca}^{2+}$-CaMK-CREB signaling pathway.

Keywords: flavonoid from Scutellaria stems and leaves ; Alzheimer's disease; Neuroregeneration; Ca ${ }^{2+}$ CaMK-CREB 


\section{INTRODUCTION}

Alzheimer's disease (AD) is a kind of neurodegenerative disease characterized by progressive cognitive handicap and memory hypomnesis. Its main pathological features include Senile plaques (Sps) formed by $\beta$-amyloid $(\mathrm{A} \beta)$ peptide deposition and neurofibrillar tangles formed by abnormal aggregation of hyperphosphorylation of tau protein ${ }^{[1]}$. At the same time, the reduction of the number of neurons and the decrease of neuroregeneration are also accompanied by the pathological process of $\mathrm{AD}{ }^{[2]}$. Therefore, promotion of neuroregeneration may become a mean of treatment of AD. CAMP-response element binding protein (CREB) is a recognized nuclear transcription factor with the function of regulating the growth, differentiation, survival and regeneration of neurons ${ }^{[3]}$. It plays an important role in the central nervous system in regulating the expression of its downstream genes through autophosphorylation. CREB phosphorylation is regulated by many signal pathways, among which $\mathrm{Ca}^{2+}$ CaMK-CREB signaling pathway is a pivotal pathway. Phosphorylation of CREB at Ser133 site is produced through the activation of calmodulin dependent protein kinaseII (CaMK II) ${ }^{[4]}$. Basic fibroblast growth factor 2 (FGF2) and Early growthresponse-1 (Egr-1) are the downstream gene expression products of $\mathrm{CREB}$, and they are important molecules to promote neuroregeneration and nerve process growth.

Studies have showed that both deposition of $\mathrm{A} \beta$ and aluminum poisoning can cause neurotoxicity, which is closely related to the occurrence of $\mathrm{AD}$. Intraventricular injection of amyloid beta protein 25$35\left(\mathrm{~A} \beta_{25-35}\right)$ in combination with aluminum trichloride $\left(\mathrm{AlCl}_{3}\right)$ and recombinant human transforming growth factor- $\beta 1$ (RHTGF- $\beta 1$ ) (composited $A \beta$ ) to establish mimic AD memory impairment model was closer to simulating the neuro-pathological states of clinical AD with multiple pathogenesis participation ${ }^{[5]}$.

SSF, extracted from the aerial parts of Scutellaria baicalensis stems and leaves, has been proved to exert antibacterial, antioxidant, anti-oxygen and improvement memory impairment ${ }^{[6-9]}$. However, it has not been reported whether the effect of SSF on the decrease of neuro-regeneration induced by composited $\mathrm{A} \beta$ in rats is regulated by $\mathrm{Ca}^{2+}-\mathrm{CaMK}-\mathrm{CREB}$ signaling pathway. In the present study, the rats' model of mimic $A D$ memory impairment was established by intraventricular injection of $A \beta_{25-35}$ in combination with $\mathrm{AlCl} 3$ and RHTGF- $\beta 1$ (composited $\mathrm{A} \beta$ ) and effect of SSF on the decrease of neuroregeneration induced by composited $A \beta$ and the effective molecule mechanism of SSF in $\mathrm{Ca}^{2+}-\mathrm{CaMK}-$ CREB signaling pathway were reported, which provided an experimental foundation to further clarify that $\mathrm{SSF}$ can treat $\mathrm{AD}$ by promoting neuro-regeneration.

\section{Materials ANd Methods}

\subsection{Experimental Materials}

\subsubsection{Animals and Instruments}

Sixty healthy adult male SD rats $(250 \pm 10 \mathrm{~g})$ were purchased from the experimental animal center of Hebei Medical University, and the certificate number is SCXK-2013-0001. They were housed in groups (five per cage) with free access to food and water, under controlled laboratory conditions for 12-hour light-dark cycle and ambient temperature of $22-24^{\circ} \mathrm{C}$. The rats were allowed to acclimatize to the laboratory environment for 7 days before the operation. All animal procedures are carried out in accordance with the "Animals management regulations" promulgated by the State Science and Technology Commission of the People's Republic of China on November 14, 1988 and revised on March 1, 2017. In addition, the less number of animals used and their discomforted should be as much as possible. 68000 Series Brain Stereotactic Instrument, Syringe Pump 200 Series and Small Animal Anaesthesia Machine RWD510 were provided by Shenzhen RWD life Science and Technology Co., Ltd. Optical microscope was purchased from OLYMPUS Company, Japan. Morris water maze was provided by Institute of Medicine, Chinese Academy of Medical Sciences. IMAGER550 gel image analysis system was supplied by ALPHA Company, America. Agilent Mx3000P QPCR and StrataGene quantitative PCR instrument were provided by American Agilent Company.

\subsubsection{Drug and Reagents}

SSF is prepared by pharmacological laboratory of Chengde Medical College (Purity $>80 \%$, Lot.20100618). A $\beta_{25-35}$ (Lot. MB10445) was provided by Dalian Meilun Company, China. AlCl3 was 
supplied by Tianjin Beichen Chemical Reagent Co, Ltd, China. Recombinant Human TGF- $\beta 1$ (Lot. 0218209-1) was purchased from PEPROTECH Company. NeuN first antibody (Lot. BST17154354) and sheep anti-rat second antibody (Lot. BST14C19B50) were purchased from BOSTER Biotechnology Co. Ltd. Rabbit monoclonal Egr-1 antibody (Lot. ab133695), Rabbit monoclonal CAMKII antibody (Lot. ab52476), Rabbit monoclonal CAMKIV antibody (Lot. ab75874) and sheep anti-rabbit second antibody (Lot. ab97051) were purchased from abcam Company, British. Rabbit polyclonal FGF2 antibody (Batch No. 4926) and Rabbit polyclonal CREB-Ser133 antibody (Lot. 9505) were supplied by SAB Company. TRIzol Reagent (Lot. 101002) was provided by ambion Company. CaMKII, FGF2, Egr-1 primer, TB Green ${ }^{\mathrm{TM}}$ Premix Ex Taq ${ }^{\mathrm{TM}}$, PrimeScript RT reagent Kit and the primer were purchased from TaKaRa Company.

\subsection{Establishment and Screening of Model}

Sixty healthy adult SD male rats were randomly divided into the sham group (10 rats) and the operated group (50 rats). The rats were treatd adaptively for one week. The operated group used the method of $\mathrm{Wu}$ xiaoguang et al. to establish the rat model of mimic AD memory impairment by intraventricular injection composited $A \beta^{[10]}$. The rats were anesthetized with isoflurane and fixed on the rat brain stereotaxic apparatus, and the skin was cut open to expose the skull. Three points were marked on the skull according to the Bao Xinming rat brain stereolocation map ${ }^{[11]}$. The bregma point was the coordinate origin, and the first point opened at $1.4 \mathrm{~mm}$ on the right side of the sagittal suture of $2.0 \mathrm{~mm}$ after the bregma point (for injection of RHTGF- $\beta 1$ ). The second point was $0.8 \mathrm{~mm}$ behind the bregma and $2.0 \mathrm{~mm}$ on the left side of sagittal suture (for injection of $\mathrm{A} \beta_{25-35}$ and $\mathrm{AlCl} 3$ ). The third point is located at $2.0 \mathrm{~mm}$ before the bregma point and $1.5 \mathrm{~mm}$ on the left side of the sagittal suture (for screw fixation). A needle connected to the syringe pump was inserted into the first hole and $1 \mu \mathrm{L}$ of RHTGF$\beta 1(10 \mathrm{ng})$ was injected into the hole. The guide casing was embedded at the second point, the first and third holes were fixed with small screws, and the denture base material and denture base water were mixed at the ratio of $1.5 \mathrm{~g} / \mathrm{ml}$ and then dumped on the plastic base of the catheter and two screws to fix the guide casing and cover the whole skin incision to avoid skin infection. From the second day after operation, the rats in the operation group were microinjected with $4 \mu \mathrm{g}(1 \mu \mathrm{L}) \mathrm{A} \beta_{25-35}$ in the morning for $14 \mathrm{~d}$ and $3 \mu \mathrm{L}$ aluminum trichloride (1\%) in the afternoon for 5 days. And the rats in the sham group underwent the same operation but were injected with the same amount of normal saline. On the 45th day after operation, the rats were trained with Morris water maze for 4 consecutive days, and the success rate of $\mathrm{AD}$ memory impairment model was calculated by using the water maze score on the 4th day. The success rate of this experiment was $83.3 \%$.

\subsection{Grouping, Administration and Sampling of Animals}

The model successful rats were randomly divided into model group and three-dose drug groups. The rats in the drug group were orally given 35,70 and $140 \mathrm{mg} / \mathrm{kg}$ SSF for 31 days, respectively. The rats in the model group and the sham group were given equal volume saline. All the rats were decapitated after the last administration of $40 \mathrm{~min}$. The right brain of three rats in each group was fixed with $4 \%$ formaldehyde for 48 hours and embedded in paraffin after routine conduct. The hippocampus and cerebral cortex were isolated on ice and wrapped in aluminum foil and placed in $1.5 \mathrm{~mL}$ Ep tubes and frozen in an ultra-cryogenic refrigerator at $-86{ }^{\circ} \mathrm{C}$ for subsequent Western blotting and qPCR examination.

\subsection{Immunohistochemical Detects of Neun Protein Expression of Hippocampal Gyrusneurons of Rats}

Paraffin sections were conventional dewaxed to water, repaired with citrate buffer solution and blocked with $5 \% \mathrm{BSA}$, and then added NeuN primary antibody, $4{ }^{\circ} \mathrm{C}$ for the night. After incubation with the second antibody, DAB was used to chromogenic reaction and neutral gum was used to seal slices. Finally, the sections were observed and photographed under 400× OLYMPUS microscope. Three slices were selected in each group, and three visual fields were randomly collected from each slice. 


\subsection{Western Blotting Detects Camk II, P-CREB-Ser133, FGF2 and Egr-1 Proteins Expression Levels in Hippocampus and Cortex of Rats}

The hippocampal and cortical tissues of each group were Grinded, and the tissue protein was extracted with RIPA lysate (containing PMSF), and the concentration of sample protein was determined by BCA method. The protein was transferred to PVDF membrane by wet transfer method after SDS-PAGE electrophoresis, then sealed with milk powder, and the first antibody of CaMK II , p-CREB-Ser133, FGF2 and Egr-1 was added separately and placed overnight at $4{ }^{\circ} \mathrm{C}$. The next day, the membrane was washed after the incubation with the second antibody, and exposed and developed with ultra-sensitive ECL chemiluminescence agent. The protein bands were analyzed by Image $J$, and the ratio of peak area of target protein to internal reference $\beta$-actin protein was used as their relative expression.

\subsection{Qpcr Detects Camk II , FGF2 and Egr-1 Mrna Expression Levels in Hippocampus and Cortex of Rats}

RNA was extracted from rat hippocampus and cerebral cortex by Trizol and the concentration of RNA and OD 260/ 280 values were detected (1.8-2.1 were qualified). The reverse transcription kit was used for reverse transcription, and the product was c DNA. As the housekeeper gene, the sequences of the upstream and downstream primer of $\beta$-actin are 5'-GGAGATTACTGCCCTGGCTCCTA-3' and 5'GACTCA -TCGTACTCCTGCTTGCTG-3', and the product size is $150 \mathrm{bp}$. The sequences of the upstream and downstream primer of CAMKII are 5'-CCATCACCAGAATGG -GACACAG-3' and 5'GCATCATGGAGGC-AACAGTAGAAC-3', the product size is 148 bp. The upstream and downstream primer sequences of FGF2 are 5'-CGAA -CCGGTACCTGGCTATGA-3' and 5'GTATTTCCGTGACCGGTAAGTGTTG-3', and the product size is $125 \mathrm{bp}$. The upstream and downstream primers of Egr-1 are 5'-GAACAACCCTACGAGCACCTG-3' and 5'-GCCACAAAGT GTTGCCACTG-3', the product size is $172 \mathrm{bp}$. The reverse transcription product c DNA was amplified with TB GreenTM Premix Ex TaqTM II and the dissolution curve of the amplified product was obtained. $2^{-\triangle \Delta C}$ method was used for relative quantitative calculation of the data, and the formula was $\mathrm{F}=2-\{$ (the average $\mathrm{Ct}$ value of the target gene in the tested group - the average $\mathrm{Ct}$ value of the house-keeping gene in the tested group) $\times$ (the average $\mathrm{Ct}$ value of the target gene in the control group - the average $\mathrm{Ct}$ value of the house-keeping gene in the control group)\}, and the transcriptional expression level of the target gene relative to the internal reference mRNA was calculated.

\subsection{Statistical Analyses}

All the data were analyzed by SPSS 19 Statistical Software, and the data was expressed as mean \pm SD. Single factor analysis of variance (one-way ANOVA) was used to compare the mean of multiple samples. $P<0.05$ was considered to be statistically significant.

\section{RESULTS}

\subsection{Effect of SSF on the Protein Expression of Neun in The Hippocampal Gyrus of the Rats}

Figure 1 is the determinative result of light microscopy of NeuN protein expression in the hippocampal gyrus of rats. The results showed that the positive expression of NeuN protein in hippocampal gyrus of rats in sham group was significantly higher, which is brownish yellow granule. Most of the staining sites were in the nucleus and a few in the cytoplasm. Compared with the sham group, the positive expression of NeuN in hippocampal gyrus cells of the model group was significantly lessened, and almost all of the staining sites were in the cytoplasm, and very few in the nucleus. However, three doses of SSF can markedly increase the protein expression of NeuN in hippocampal gyrus induced by composited $A \beta$ to varying degrees. Compared with the model group, the positive expression of NeuN in rats' hippocampal gyrus in 35, 70 and $140 \mathrm{mg} / \mathrm{kg}$ SSF groups was reversed the decrease induced by composited $\mathrm{A} \beta$. The most of the staining sites were in cytoplasm, the staining of nucleus increased successively, and the staining color deepened. 

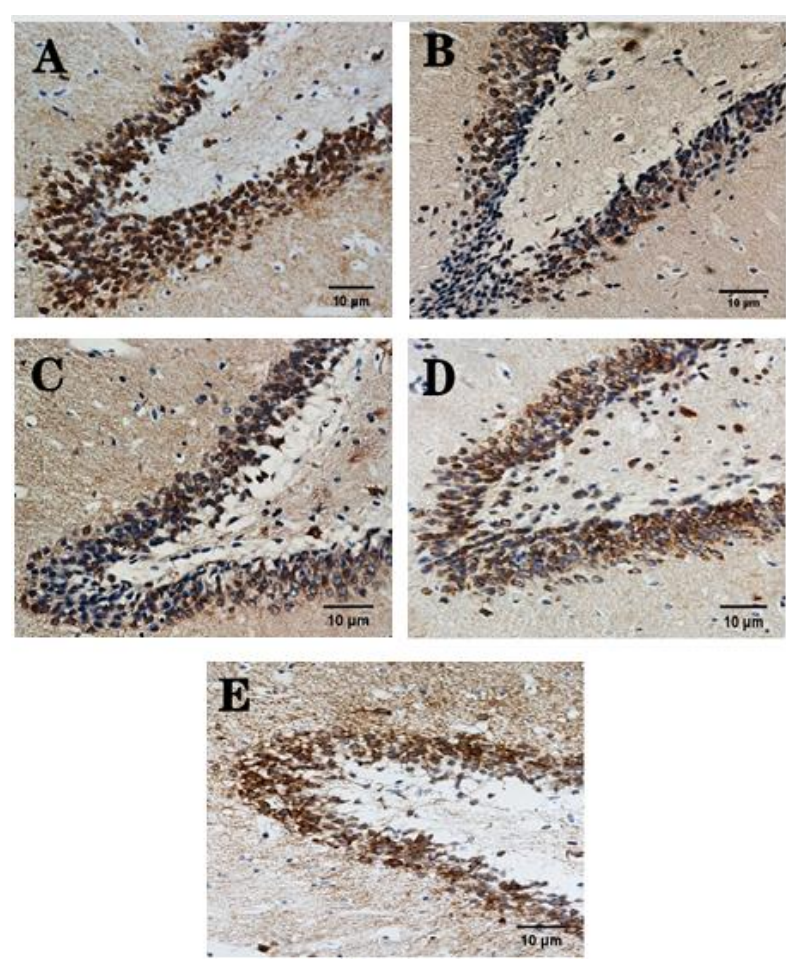

Figure1. The Effect of SSF on the expression of NeuN in the Hippocampal gyrus of the composited A $\beta$ rats. A: sham group; B: model group; C: SSF 35mg/kg group; D: SSF70mg/kg group; E: SSF70mg/kg group.

\subsection{Effect of SSF on the Expression of Camk II Proteins in Hippocampus and Cerebral Cortex of Rats}

Figure 2 shows that compared with sham group, the content of CaMKII protein in hippocampus and cerebral cortex of model group rats was reduced by $90.23 \%(\mathrm{P}<0.01)$ and $182.07 \%(P<0.01)$, respectively. However, three doses of SSF can regulate the CaMKIV protein expression level in hippocampus and cerebral cortex of rats induced by composited $A \beta$ to varying degrees. Compared with the model group, 35 and $140 \mathrm{mg} / \mathrm{kg}$ SSF increased the content of CaMKII protein in hippocampus of rats with composited $\mathrm{A} \beta$ by $10.34 \%$ and $22.83 \%$ respectively $(P<0.01)$. Three doses of SSF increased the content of CaMKII protein in the cerebral cortex by $95.77 \%, 105.24 \%$ and $148.41 \%$, respectively $(P<0.01)$.

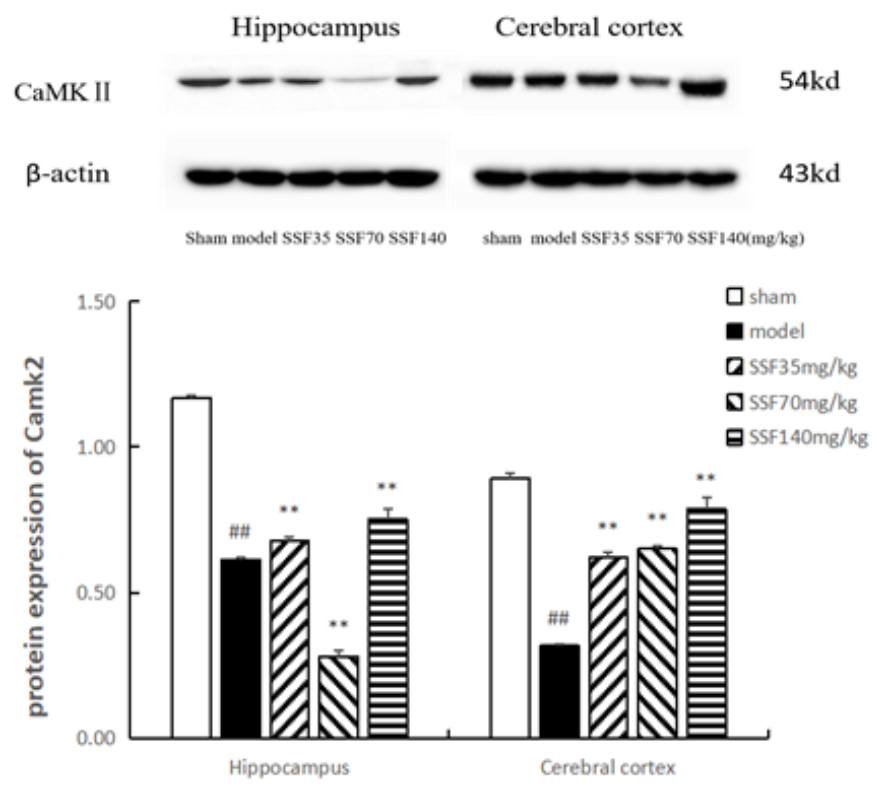

Figure2. The effect of SSF on the expression of CaMK II proteins in hippocampus and cerebral cortex of rats with composited AB. Mean $\pm S D . \quad n=3 .{ }^{\# p} p<0.01$ vs sham group; ${ }^{* *} p<0.01$ vs model group. 


\subsection{Effect of SSF on the Protein Expression of P-CREB-Ser133 in the Hippocampus and Cerebral Cortex of Rats}

Figure 3 shows that the protein content of P-CREB-Ser133 in the hippocampus and cerebral cortex of the model group decreased by $84.87 \%$ and $12.50 \%(P<0.01)$, respectively, as compared with the sham group. However, the three doses of SSF can regulate the p-CREB-Ser133 protein expression level in hippocampus and cortex of rats induced by composited $A \beta$ to varying degrees. Compared with the model group, 70 and $140 \mathrm{mg} / \mathrm{kg}$ SSF increased the content of $p$-CREB-Ser133 protein in the hippocampus by $22.57 \%$ and $24.97 \%(P<0.01)$, respectively, and three doses of SSF increased the content of p-CREB-Ser133 protein in the cortex by $48.25 \%, 47.38 \%$ and $2.90 \%(P<0.01, P<0.05)$, respectively.

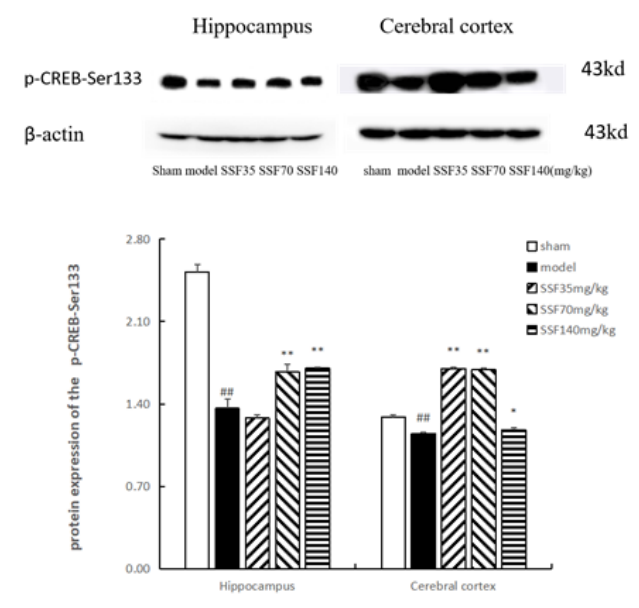

Figure3. The effect of SSF on the expression of p-CREB-Ser133 protein in hippocampus and cerebral cortex of rats with composited AB. Mean $\pm S D . \quad n=3$. ${ }^{\#} p<0.01$ vs sham group; ${ }^{* *} p<0.01$ vs model group, ${ }^{*} p<0.05$ vs model group.

\subsection{Effect of SSF on the Protein Expression of FGF2 in the Hippocampus and Cerebral Cortex of Rats}

Figure 4 shows that the FGF2 protein content in the hippocampus and cerebral cortex of the model group decreased by 1.63 times $(P<0.01)$ and 1.55 times $(P<0.01)$, respectively, as compared with the sham group. However, three doses of SSF can increase the FGF2 protein expression level in hippocampus and cerebral cortex of rats induced by composited $A \beta$ to varying degrees. Compared with the model group, three doses of SSF increased FGF2 protein content in the hippocampus by $62.02 \%$, $62.02 \%$ and $133.03 \%(P<0.01)$, respectively, $70 \mathrm{mg} / \mathrm{kg} \mathrm{SSF}$ increased the content of FGF2 protein in the cerebral cortex of composited $\mathrm{A} \beta$ rats by $25.71 \%(\mathrm{P}<0.01)$, respectively.
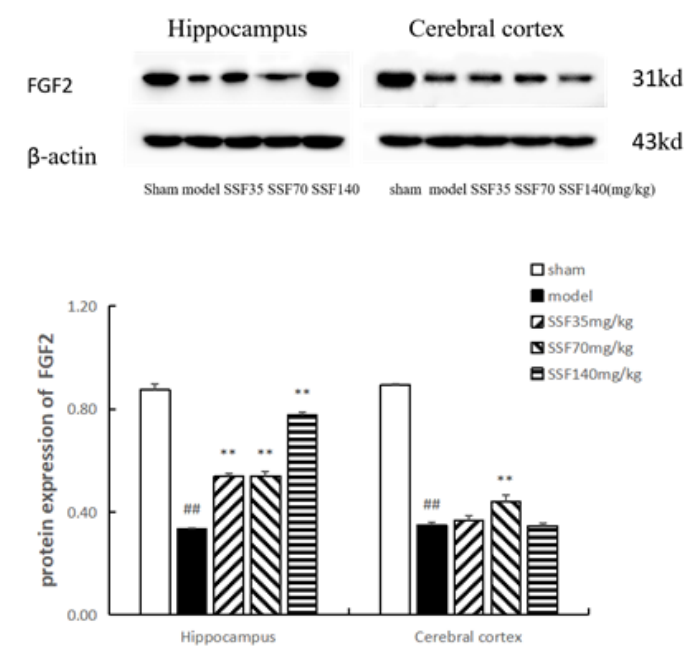

Figure4. The effect of SSF on expression of FGF2 protein in hippocampus and cerebral cortex of rats in each group. Mean $\pm S D . n=3$. ${ }^{\#} p<0.01$ vs sham group; ${ }^{* *} p<0.01$ vs model group. 


\subsection{Effect of SSF on the Protein Expression of Egr-1 In The Hippocampus and Cerebral Cortex of Rats}

Figure 5 shows that compared with the sham group, the content of Egr-1 protein in the hippocampus and cortex of the model group decreased by $141.10 \%$ and $80.03 \%(P<0.01)$, respectively. Three doses of SSF can increase the Egr-1 protein expression level in hippocampus and cortex of rats induced by composited $A \beta$ to varying degrees. Compared with the model group, three doses of SSF increased the content of Egr-1 protein in the hippocampus of composited $\mathrm{A} \beta$ rats by $250.00 \%, 183.33 \%$ and $181.10 \%$ $(P<0.01)$, respectively, and the content of Egr-1 protein in the cerebral cortex increased by $75.22 \%$, $182.05 \%$ and $28.40 \%(P<0.01)$, respectively.

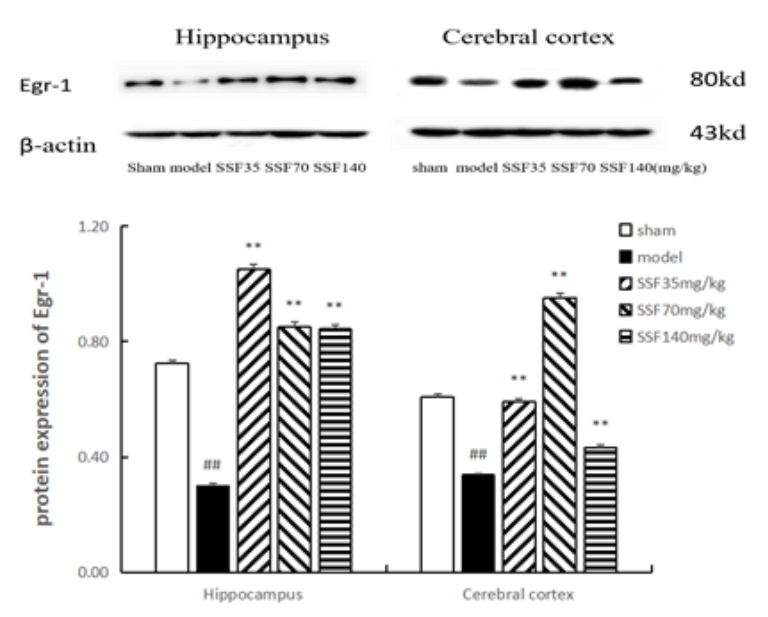

Figure5. The effect of SSF on the expression of Egr-1 protein in the hippocampus and cerebral cortex of rats in each group. Mean $\pm S D . n=3$. ${ }^{\# \#} p<0.01$ vs sham group; ${ }^{* *} p<0.01$ vs model group.

\subsection{Effect of SSF on the Expression of Camk II Mrna in Hippocampus and Cerebral Cortex of Rats}

Figure 6 is the result of CaMKII in hippocampus and cerebral cortex of rats by qPCR. The results showed that the content of CaMK II mRNA in hippocampus and cerebral cortex of the model group was $47.25 \%$ and $190.78 \%(P<0.01)$ lower than those of the sham group, respectively. However, three doses of SSF can regulate the CaMK II mRNA expression level in hippocampus and cerebral cortex of rats induced by composited $A \beta$ to varying degrees. Compared with model group, 70 and $140 \mathrm{mg} / \mathrm{kg}$ SSF increased CaMK II mRNA expression in the hippocampus by $36.78 \%$ and $65.87 \%(P<0.01)$ respectively, and $35 \mathrm{mg} / \mathrm{kg}$ SSF increased CaMK II mRNA expression in the cerebral cortex by $138.41 \%$ $(P<0.01)$.

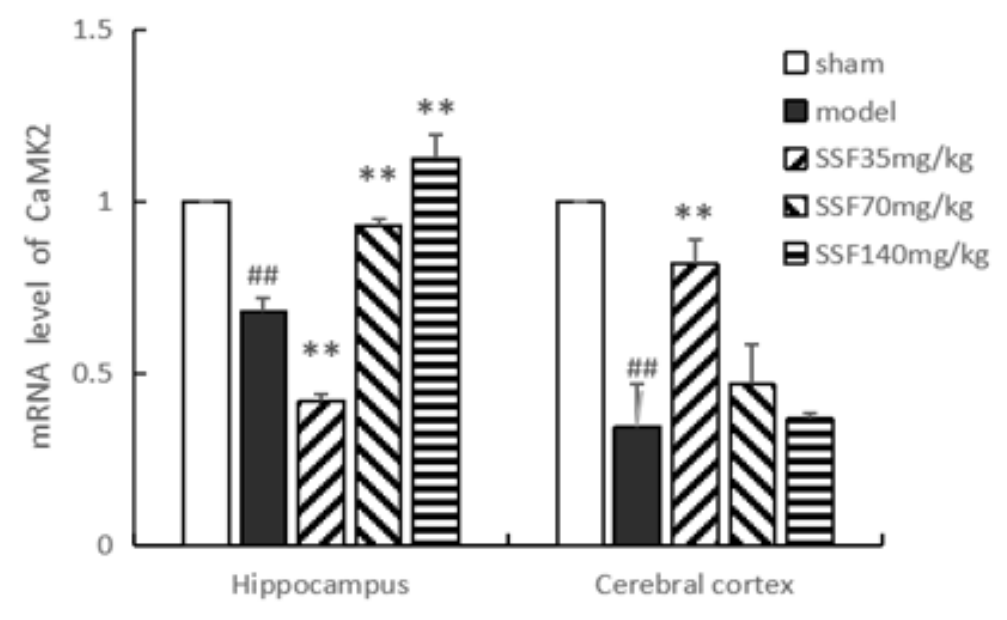

Figure6. The Effect of SSF on the expression of CaMK II mRNA in hippocampus and cerebral cortex of rats with composited AB. Mean $\pm S D . n=3$. ${ }^{\#} p<0.01$ vs sham group; ${ }^{* *} p<0.01$ vs model group. 


\subsection{Effect of SSF on the Expression of FGF2 Mrna in Hippocampus and Cerebral Cortex of Rats}

Figure 7 is the result of FGF2 in hippocampus and cortex of rats by qPCR. The results showed that compared with the sham group, the expression of FGF2mRNA in the hippocampus of the model group increased by $22.74 \%(P<0.05)$. However, three doses of SSF could regulate the expression level of FGF2mRNA in hippocampus / cerebral cortex of rats induced by composited $\mathrm{A} \beta$ to varying degrees. Compared with the model group, 70 and $140 \mathrm{mg} / \mathrm{kg}$ SSF increased the expression of FGF2mRNA in cortex of rats with composited $\mathrm{A} \beta$ by $38.45 \%(P<0.01)$ and $13.78 \%$, respectively.

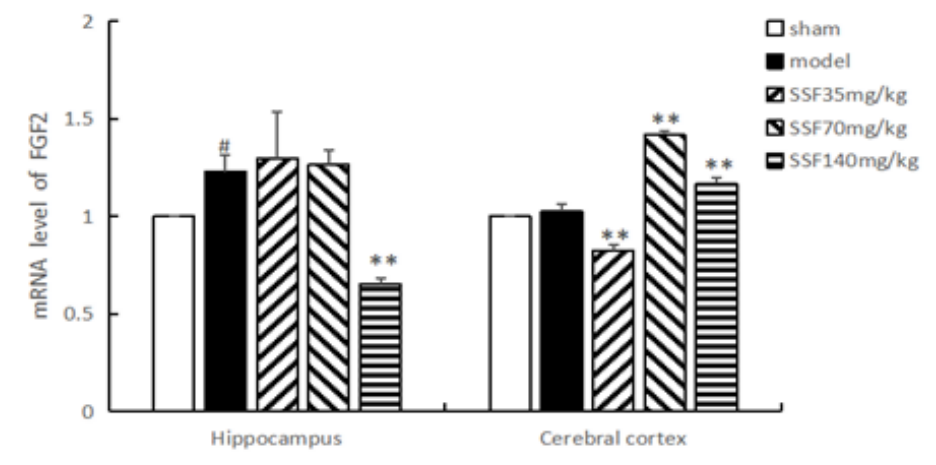

Figure7. The effect of SSF on the expression of FGF2 mRNA in hippocampus and cerebral cortex of rats with composited AB. Mean $\pm S D . \quad n=3 .{ }^{\#} p<0.05$ vs sham group; ${ }^{* *} p<0.01$ vs model group.

\subsection{Effect of SSF on the Expression of Egr-1 Mrna in Hippocampus and Cerebral Cortex of Rats}

Figure 8 is the result of Egr-1 in hippocampus and cerebral cortex of rats by qPCR. Compared with the sham group, the expression of Egr-1 mRNA in the cortex of the model group decreased by $274.95 \%$ $(\mathrm{P}<0.01)$. However, three doses of SSF can increase the expression level of Egr-1 mRNA in hippocampus and cerebral cortex of rats induced by composited $A \beta$ to varying degrees. Compared with the model group, 35 and $70 \mathrm{mg} / \mathrm{kg}$ SSF increased the expression of Egr-1 mRNA in hippocampus of rats with composited $\mathrm{A} \beta$ by $194.01 \%$ and $86.91 \%$ respectively $(\mathrm{P}<0.01) .35 \mathrm{mg} / \mathrm{kg}$ SSF increased the expression of Egr-1 mRNA in cerebral cortex of rats with composited A $\beta$ by $240.64 \%(\mathrm{P}<0.01)$, respectively.

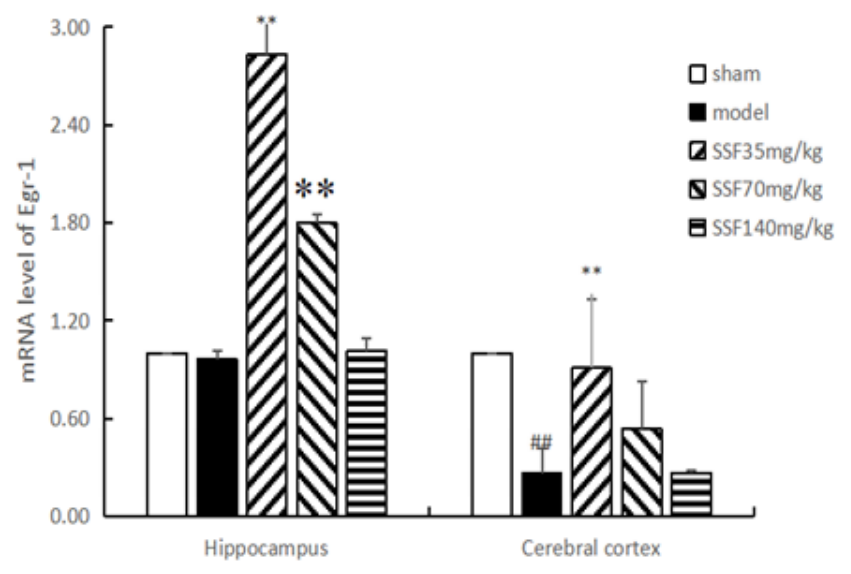

Figure8. The effect of SSF on the expression of Egr-1 mRNA in hippocampus and cerebral cortex of rats with composited $A \beta$. Mean $\pm S D$. $n=3$. ${ }^{\# \#} p<0.05$ vs sham group; ${ }^{* *} p<0.01$ vs model group.

\section{DISCUSSION}

$\mathrm{AD}$ is a progressive neurodegenerative disease with hidden onset, which is pathologically manifested as SPs formed by extracellular A $\beta$ deposition, NFTs formed by intracellular tau protein hyperphosphorylation, neuronal degeneration and loss, reduction of neurotransmitters, glial cell proliferation and vascular amyloidosis ${ }^{[12]}$. The occurrence and development of AD is accompanied by 
the decrease of neuroregeneration, and the decrease of neuroregeneration further aggravates the cognitive impairment of AD patients ${ }^{[13]}$. Promoting neuroregeneration may be a compensatory response to compensate for the pathological deficiency and neurological function to AD. Neuroregeneration was promoted which can increase synaptic plasticity, repair damaged neural circuits and restore cognitive function. Under normal circumstances, neural precursor cells can produce new neurons and develop into mature cells with neural functions. However, for $\mathrm{AD}$ patients, although the neural stem cells in the subgranule lower area of the dentate gyrus of the hippocampus can proliferate and differentiate, they cannot develop into mature neurons ${ }^{[14]}$. Therefore, $\mathrm{AD}$ can be evaluated by detecting the number of mature nerve cell elements in the brain. Neuronal core antigen neuron, a specific protein NeuN is a soluble protein distributed in mature nerve nucleus of vertebrate nervous system. NeuN can specifically bind to the nucleus and cytoplasm of mature nerve cells, and has the characteristics of high conservation in vivo and stable expression during the special stage of nerve cell growth ${ }^{[13]}$. It is often used as a specific marker of mature nerve cells and an important target of neural structure and function. In this study, it was found that intraventricular injection of composited $A \beta$ significantly reduced the expression of NeuN in the hippocampal gyrus of rats. The results showed that intraventricular injection of composited $A \beta$ can reduce the number of mature neurons, and the results were parallel to the decrease of neuroregeneration in patients with AD. However, three doses of SSF can reverse the decrease of NeuN in rat hippocampal gyrus neurons induced by composited $A \beta$ to varying degrees, which indicates that SSF can increase the number of mature neurons in the brain of AD-like rats and promote neuroregeneration.

In the process of neuroregeneration, multiple protein factors are involved in the survival and integration of new neurons. CREB is one of the most important protein factors, which can regulate the key steps of neuroregeneration, such as cell survival, proliferation, differentiation, growth and development, as well as the synaptic plasticity and long-term memory formation of nerve cells ${ }^{[15]}$. The biological activity of the CREB protein is regulated by the phosphorylation of the Ser-133 site in vivo, while the phosphorylation of CREB is regulated by several signaling pathways including CAMP-CREB, $\mathrm{Ca}^{2+}$ CaMK-CREB, Ras-ERK-CREB and P13-Akt-CREB ${ }^{[16]}$. Extracellular signaling causes intracellular $\mathrm{Ca}^{2+}$ to bind with calmodulin $(\mathrm{CaM})$ to form bioactive calcium-calmodulin complex $\left(\mathrm{Ca}^{2+} / \mathrm{CaM}\right)$. through the $\mathrm{Ca}^{2+}$-CaMK-CREB signaling pathway. $\mathrm{Ca}^{2+} / \mathrm{CaM}$ further activated calmodulin kinase II . Activated CaMK II can phosphorylate Ser-133 site of CREB, p-CREB-Ser133 promoted neuroregeneration by activating its downstream protein factor ${ }^{[17]}$. Studies have found that CaMK II is a kind of polymerase enzyme, which widely exists in nerve tissue, is an important component of mammalian forebrain total protein and postsynaptic dense part ${ }^{[18]}$, and It is also the key regulator of synaptic plasticity. The increase of CaMKII can increase the plasticity of synapses and promote neuroregeneration. The increase of CaMKII can increase the plasticity of synapses and promote neuroregeneration. Therefore, the activation of CaMKII and CREB can accelerate the proliferation and morphological maturation of neonatal neurons. The present study found that intraventricular injection of composited A $\beta$ can significantly decrease the expression of CaMK II and p-CREB-Ser133 protein in the brain of rats. And three-doses SSF could reverse the decrease of CaMK II and p-CREB-Ser133 expression in hippocampus and cerebral cortex of rats to varying degrees. These results suggested that SSF can promote neuroregeneration by increasing the proteins content of CaMKII and p-CREB-Ser133 in the brain of rats with $\mathrm{AD}$-like disorder.

In the process of neuroregeneration, CREB is the key executor of cell survival mediated by neurotrophic factor ${ }^{[19]}$. Nerve growth factor is regulated by p-CREB-Ser133 and plays an important role in cell survival. Nerve growth factor has the function of promoting nerve tissue injury repair, nerve protection and nerve nutrition ${ }^{[20]}$. The increase of its expression can promote neuroregeneration and further improve nerve function including AD memory impairment. FGF2 and Egr-1 are two important neurotrophic factors related to neuroregeneration.

FGF2 is an effective neurotrophic factor that can promote neuronal regeneration and nervous process growth, and maintain the survival of cortical neurons. It is involved in the growth and differentiation of a variety of neurons and can stimulate the mitosis and differentiation of neuron progenitor cells and glial cells ${ }^{[21]}$. It has been showed that on the one hand, FGF2 plays a role by regulating CREB to activate cyclic adenosine monophosphate (cyclic adenosine 3', 5'-monophosphate, cAMP) to induce the growth and differentiation of nerve cells ${ }^{[22]}$. On the other hand, FGF2 accelerates the metabolic renewal of 
neurons by regulating the calcium channels of the cell membrane ${ }^{[23]}$. Moreover, FGF2 can promote the survival of various cells in the central nervous system, such as VZ cells in the embryonic cerebral cortex, and can regulate the neuronal density and change the cell structure in the middle cerebral cortex. This experiment found that intraventricular injection of composited $\mathrm{A} \beta$ can decrease the protein content of FGF2 in rats. However, three-dose SSF can reverse the decrease of FGF2 protein expression and regulate the expression of FGF2 mRNA in the brain of rats induced by composited A $\beta$. This result suggests that SSF can promote neuroregeneration by increasing the protein content of FGF2 in the brain of rats with quasi-AD.

Egr-1 is known as a nerve growth factor inducible factor, and it is a member of a family of immediate early genes (IEGs). It is a downstream gene of CREB and is regulated by it. Phosphorylated CREB binds to cAMP response element (CRE) and can induce Egr-1 transcription. Activated Egr-1 is closely related to synaptic plasticity, learning and memory, and neuroregeneration ${ }^{[24]}$. It is an important molecule in the process of synaptic plasticity and the formation, consolidation and re-consolidation of long-term memory. In this study, it was found that intraventricular injection of composited $A \beta$ can decrease the expression of Egr-1 protein and mRNA in the brain of rats. However, three-doses SSF could reverse the decrease of Egr-1 protein and mRNA expression in hippocampus and cerebral cortex of rats. This suggests that SSF can promote neuroregeneration by increasing the expression of Egr-1 protein and mRNA in the brain of rats with quasi-AD. These results showed that SSF has a different dose-effect relationship with the expression of CaMK II , P-CREB-Ser133, FGF2 and Egr-1 proteins and mRNA in the hippocampus and cerebral cortex of the composited $\mathrm{A} \beta$ rats. The reason may be that flavonoids from stems and leaves of Scutellaria baicalensis is a kind of mixture in which different components interact with each other.

Precipitation-type $A \beta$ is the main component of senile plaques in the cerebral cortex and is an important factor in causing AD. Aluminum can inhibit the transformation of precipitated $A \beta$ to dissolved $A \beta$, and RHTGF- $\beta 1$ can enhance the deposition of $A \beta$ in the brain ${ }^{[25]}$. The nerve injury induced by the three can simulate the characteristics of human AD more comprehensively. The results showed that intraventricular injection of $A \beta_{25-35}$ in combination with $A \mathrm{All}_{3}$ and RHTGF- $\beta 1$ could reduce the protein contents of CaMK II , p-CREB-Ser133, FGF2 and Egr-1 in the brain, and then induce the decrease of neuroregeneration in the brain of rats. However, SSF can regulate the mRNA contents of CaMK II, FGF2 and Egr-1 in varying degrees, increase the expression of CaMK II , p-CREB-Ser133, FGF2 and Egr-1 proteins in hippocampus and cerebral cortex, and reverse the decrease of neuroregeneration induced by composited $A \beta$ in the brain of rats. The mechanism may activate $\mathrm{Ca}^{2+}$-CaMK-CREB signaling pathway by up-regulate the expression of CaMKII, increase the phosphorylation of CREB, and further increase the expression levels of neurotrophic factors FGF2 and Egr-1 of downstream of CREB. The present study suggested that the effect of SSF on neuroregeneration may be beneficial to treatment of $\mathrm{AD}$.

\section{AUTHORS CONTRIBUTION}

Shang Yazhen conceived and designed the experiment. Zhang Hui, Ding Shengkai, Liu Qianqian and Ye Yuanyuan conducted the test and wrote the manuscript.

\section{ACKNOWLEDGEMENT}

The authors wish to thank Hebei Provincial Education Department (No. ZD20131022, ZD2019057), Hebei Provincial Hundred Outstanding Innovated Talents, First Batch of China and the Key Subject Construction Project of Hebei Provincial College of China for financial support.

\section{REFERENCES}

[1] Zhao Y, Dua P, Lukiw WJ. Microbial sources of amyloid and relevance to amyloidogenesis Alzheimer's Disease(AD) . Alzheimer and Parkinson's Disease, 2015, 5(1):177 184.

[2] Chen L. Research progress on the relationship between aging and nerve regeneration of hippocampal nerve cells and Alzheimer's disease. Hebei traditional Chinese medicine, 2017, 39(10): 1583-1587.

[3] Lonze BE, Riccio A, Cohen S, et al. Apoptosis, axonal growth defects, and degeneration of peripheral neurons in mice lacking CREB. Neuron, 2002;34(3): 371-85.

[4] Guo CP, Wei Z, Huang F, et al. High salt induced hypertension leads to cognitive defect. Oncotarget, 2017 , 8(56): 95780-95790. doi: 10.18632/oncotarget. 21326. 
[5] Guo K, Wu XG, Cui YD, et al. Regulation and mechanism of Scutellaria barbata flavonoids on apoptosis of cortical neurons and cytochondriome induced by composite A $\beta$. Chinese Journal of Hospital Pharmacy, 2015, 35(22): 1994-1999.

[6] Wang RT, Zhang JX, Dong YJ. Protective effect of scutellaria baicalensis stem-leaf total flavonoid against hippocampus neurodamage induced by injection A $325-35$. Chinese Journal of Gerontology, 2010, 30(7): 926928.

[7] Shang YZ, Su BF, Wang YL. Advances in pharmacology of root, stem and leaf components of scutellaria baicalensis. Journal of Chengde Medical College, 2005(02): 153-155.

[8] Shang YZ, Meng YB, Miao H, et al. Anti-hypoxic effects of SSF on brain in mice. Chinese Journal of traditional Chinese medicine information, 2002,9(7): 24-25.

[9] Shang YZ, Gong MY, Zhou XX, et al. Improving effects of SSF on memory deficits and pathological changes of neural and immunological systems in senescent mice. Acta pharmacologica Sinica, 2001, 22(12): 10781083.

[10] Shang YZ, W XG, Cheng JJ, Zhang H. Establishment of a valuable mimic of Alzheimer's Disease in rat animal model by intracerebroventricular injection of composited amyloid Beta Protein. Journal of neuropharmacology, 2018, 8(06): 24-25.

[11] Bao XM, Si SY. Stereotactic map of rat brain. Beijing: People's Health Publishing House. 1991.

[12] Risacher SL, Anderson WH, Charil A, et al. Alzheimer disease brain atrophy subtypes are associated with cognition and rate of decline. Neurology. 2017; 89(21): 2176-2186.

[13] Duan W, Zhang YP, Hou Z, et al. Novel insights into NeuN: From neuronal marker to splicing regulator. Mol Neurobiol, 2016, 53:1637-1647.

[14] Jose J Rodriguez, Victoria C Jones, M Tabuchi, et al. Impaired adult neurogenesis in the dentate gyrus of a triple transgenic mouse model of Alzheimer's disease. PLoS One, 2008, 3(8): e2935.

[15] Lu XM, Zhu ZP, Sun LC. Research progress of CREB transcription factor phosphorylation signaling pathway and epilepsy. Chinese experimental diagnostics, 2019, 23(08): 1462-1465.

[16] Ge J, Zhang Y, Zheng ZZ. Research Progress on CREB and the signal transduction pathways of its Phosphorylation. Anhui agricultural science, 2010, 38(30): 16769-16771+16774.

[17] Wang XM. Study on the mechanism of tongqiao huoxue decoction on vascular dementia rats based on $\mathrm{Ca}^{+}-$ CaMK II -CREB pathway. Hefei: Anhui Medical University, 2016.

[18] Wang TT, Chen ZC, Ye X. Structure of calcium/calmodulin-dependent protein kinase type II and its role in nervous system. Journal of anatomy,2019, 50(03): 395-399.

[19] Tian JH, Wang XM, Han JS. Transcriptional regulation by CREB and proteins of CREB family. Progress in Physiology, 1996(03): 227-232.

[20]Dai YF, Wang TT, Ma W. Roles of nerve growth factor and brain-derived neurotrophic factor and their precursors in nervous system. Research on Organization Engineering in China, 2018, 22(28): 4580-4586.

[21] Wang SJ. Biological activity and application of basic fibroblasts growth factor. Chinese Journal of Hospital Pharmacy, 2013, 33(13): 1081-1083.

[22] Shiyong W, Wenhua Z, Shumin M, et al. ERK1/2 and JNK signalingmodulate mitogenic effect of fibroblast growth factor 2 on liver cell. Cell Biolint, 2018, 3(15): 110-130.

[23] Maric D, Rehacek J, Hradil Z, et al. Prospective cell sorting of embryonic rat neural stem cells and neuronal and glial progenitors revealsselective effects of basic fibroblast growth factor and epidermal growth factor on self-renewal and differentiation. Journal of Neuroscience, 2003, 23(1): 240-251.

[24] Koldamova R, Schug J, Lefterova M, et al. Genome-wide approaches reveal Egr-1-controlled regulatory networks associated with neurodegeneration. Neurobiology of disease, 2014, 63:107-114.

[25] Shang YaZ.Effects and mechanism of Sculellaria barbata flavonoids on rat's memory impairment induced by compound A $\beta 25-35$. WuHan University, 2013.

Citation: Shang Yazhen, et.al., (2019). Ca2+-Camk-CREB Mediates the Effect of Flavonoids from Scutellaria Baicalensis Stems and Leaves on the Decrease of Neuroregeneration-Induced by Composited AB in Rats. International Journal of Medicinal Plants and Natural Products (IJMPNP), 5(4), pp.12-22. http://dx.doi.org/10.20431/2454-7999.0504003

Copyright: () 2019Authors, this is an open-access article distributed under the terms of the Creative Commons Attribution License, which permits unrestricted use, distribution, and reproduction in any medium, provided the original author and source are credited. 